\title{
Dihydrolipoic Acid Conjugated Carbon Dots Accelerate Human Insulin Fibrillation
}

\begin{abstract}
Keywords: Protein fibrillation; Insulin; Carbon dots; Nanoparticles Abstract

Protein fibrillation is believed to play an important role in the pathology and development of several human diseases, such as Alzheimer's disease, Parkinson's disease and type 2 diabetes. Carbon dots (CDs), as a new type of nanoparticle have recently been extensively studied for potential biological applications, but their effects on protein fibrillation remain unexplored. In reality, any application in biological systems will inevitably have "contact" between proteins and CDs. In this study, human insulin was selected as a model protein to study the effects of CDs on protein fibrillation, as proteins may share a common mechanism to form fibrils. Hydrophobic CDs were conjugated with dihydrolipoic acid (DHLA-CDs) to facilitate their water solubility. Characterizations from thioflavin T fluorescence, circular dichroism spectroscopy and atomic force microscopy demonstrate that the presence of DHLA-CDs results in a higher rate of human insulin fibrillation, accelerating the conformational changes of human insulin from a-helix to $\beta$-sheet. This promoting effect is likely associated with the locally increased concentration of human insulin adsorbed on the surface of DHLA-CDs.
\end{abstract}

\section{Abbreviations}

AFM: Atomic Force Microscopy; CDs: Carbon Dots; DHLACDs: Dihydrolipoic Acid-conjugated Carbon Dots; QDs: Quantum Dots; TEM: Transmission Electron Microscopy; ThT: Thioflavin T

\section{Introduction}

Formation of amyloid-like protein fibrils with cross- $\beta$-sheet structures from protein native conformation has been indicated to play an important role in the development of several serious human diseases, such as Alzheimer's disease, Parkinson's disease, prion diseases, and type 2 diabetes mellitus [1,2]. In spite of protein diversity in sources, sequences and functions, protein fibrils share strikingly similar characteristics, including nucleation-dependent kinetics, cross- $\beta$-sheet structures, elongated morphology, and thioflavin $\mathrm{T}$ (ThT) fluorescence enhancement [1,3-5]. These observations indicate that the fibrillation of proteins may share a common molecular mechanism. Therefore, study of the protein fibrillation process may provide useful information and insights into the pathology, prevention and treatment of the human diseases associated with protein fibrillation.

Withrecentnanotechnologicaldevelopmentsinnanomedicineand biomedical engineering, much attention is focused on understanding the interaction and toxicity of nanomaterials on cellular components, such as proteins, plasma membranes and DNA [6-10]. Quantum dots (QDs), a class of typical nanoparticles, have been extensively studied with proteins to explore their potential biomedical applications. One study illustrated that $\mathrm{N}$-acetyl-L-cysteine capped CdTeQDs inhibit amyloid beta 1-40 fibril formation in vitro [11]. On the contrary,
Journal of

Parkinson's disease \& Alzheimer's disease

Sheba J. Kuruvilla ${ }^{1}$, Shanghao Li $^{1}$, Lorenzo Sansalone ${ }^{1}$, Blake Fortes ${ }^{1}$, lan Zheng ${ }^{1}$, Patrica L. Blackwelder 2,3, Cyrus Pumilia1, Miodrag Micic ${ }^{4,5}$, Jhony Orbulescu ${ }^{5}$ and Roger M. Leblanc ${ }^{1 *}$

${ }^{I}$ Department of Chemistry, University of Miami, USA

${ }^{2}$ University of Miami Center for Advanced Microscopy and Marine Geosciences, University of Miami, USA

${ }^{3}$ Nova Southeastern University Oceanographic Center, USA ${ }^{4}$ Department of Engineering Design Technology, Cerritos College, USA ${ }^{5} \mathrm{MP}$ Biomedicals LLC, USA

${ }^{*}$ Address for Correspondence

Roger M. Leblanc, Professor, Department of Chemistry, University of Miami, 1301 Memorial Drive, Coral Gables, Florida, 33146, USA, Tel: +1305-284-2194; Fax: +1-305-284-6367; E-mail: rml@miami.edu

Submission: 23 January, 2015

Accepted: 16 March, 2015

Published: 20 March, 2015

other research suggested that hydrophilic coated QDs accelerate the fibrillation of proteins (i.e., human $\beta 2$-microglobulin, $\alpha$-synuclein and human serum albumin) by enhancing the formation of the critical nucleus [12,13]. Our current knowledge of nanoparticles influencing protein fibrillation still relies on a case-by-case approach, depending on physical/chemical properties of proteins and nanoparticles, such as components, charge, coating and size $[12,14,15]$.

Another novel type of nanoparticle-carbon dots (CDs) has recently arisen to replace QDs for biological applications. Compared with QDs, CDs are currently attracting more and more research attention because of their unique properties, such as excitation wavelength dependent photoluminescence, excellent biocompatibility, low cytotoxicity, and optical stability [16-18]. These properties make CDs increasingly attractive for applications in biological systems. Several studies using CDs in biological systems have shown that CDs are nontoxic and can be used for imaging in cellular environment $[16,19,20]$. For any application of CDs in a biological system, they are inevitably interacting with proteins which may undergo conformational changes to form fibrils due to the presence of CDs. However, the effect of CDs on protein fibrillation has not been reported until most recently (March 2015) from our group [21]. In that study, water soluble CDs with size less than $6 \mathrm{~nm}$ are prepared by oxidizing carbon nano powder and these CDs are demonstrated to inhibit human insulin fibrillation in vitro in a concentration-dependent manner.

Insulin fibrils or plaques have been observed in vivo in patients suffering from diabetes after insulin infusion or repeated injection [22,23]. Insulin fibrils can be easily formed in vitro under certain condition, such as high concentrations, low $\mathrm{pH}$ and high temperatures $[3,24]$. In this study, human insulin was chosen to study the effects of CDs on its fibrillation. It is worth noting that the CDs used in this study have completely different nature from those we reported most recently [21] in terms of size, surface properties and components. The CDs in the present study have a hydrophobic core capped by dihydrolipoic acid (DHLA-CDs) to increase their water 
Citation: Kuruvilla SJ, Li S, Sansalone L, Fortes B, Zheng I, et al. Dihydrolipoic Acid Conjugated Carbon Dots Accelerate Human Insulin Fibrillation. J Parkinsons Dis Alzheimer Dis. 2015;2(1): 7.

solubility. Furthermore, the size of DHLA-CDs is much larger than $6 \mathrm{~nm}$. Different concentrations of these DHLA-CDs were added to human insulin to study the kinetics of its fibrillation. These samples were subsequently characterized by fluorescence spectroscopy of thioflavin $\mathrm{T}$ and circular dichroism spectroscopy. The morphology of fibrils was monitored visually by atomic force microscopy (AFM). Our investigation demonstrates that the presence of DHLA-CDs promotes human insulin fibrillation.

\section{Experimental Section}

\section{Materials}

Recombinant human insulin, $( \pm)$ - $\alpha$-lipoic acid (also known as DL-thioctic acid), 1-octadecene and thioflavin T wereobtained from MP Biomedicals, LLC (Solon, OH). 1-Hexadecylamine, citric acid and other chemicals were reagent grade and purchased from SigmaAldrich (St. Louis, MO). The deionized water was obtained from a Modulab 2020 water purification system (San Antonio, TX).

\section{Synthesis of hydrophobic carbon dots}

Hydrophobic carbon dots were synthesized with the procedure developed by Wang et al. [25]. A mixture of 15 mLof1-octadecene and $1.5 \mathrm{~g}$ of1-hexadecylamine was heated in a three-neck flask under argon flow to $300^{\circ} \mathrm{C}$, and then $1 \mathrm{~g}$ of citric acid was injected into the mixture. The solution was kept at $300{ }^{\circ} \mathrm{C}$ for 3 hours. After reaction, it was cooled down in air to room temperature. The solid form of hydrophobic carbon dots was obtained and purified by precipitating with acetone in an ice bath. It was further characterized by UV-vis absorption (Shimadzu UV-2600, Japan), fluorescence (Fluorolog-3, Horiba JobinYvon, Edison, NJ), Fourier transform infrared spectroscopy (PerkinElmer, Frontier), and atomic force microscopy (Agilent 5420, Santa Clara, CA).

\section{Synthesis of DHLA conjugated carbon dots (DHLA-CDs)}

Dihydrolipoic acid (DHLA) was synthesized by reducing $( \pm)$ - $\alpha$-lipoic acid with sodium borohydride in an ice bath and the product was extracted by chloroform using the same procedures developed by Clapp et al. [26]. $0.2 \mathrm{~g}$ of as-prepared hydrophobic carbon dots and $1 \mathrm{~g}$ of DHLA were added into a1 $2 \mathrm{~mL}$ mixture consisting of methanol and chloroform $(1: 1, \mathrm{v} / \mathrm{v})$, and heated at $60^{\circ} \mathrm{C}$ for 4 hours. Potassium tert-butoxide was added to basify the solution and the mixture was centrifuged at $2500 \mathrm{rpm}$ for $15 \mathrm{~min}$ to obtain precipitates, which were water soluble DHLA conjugated carbon dots (DHLA-CDs). The product was further characterized by spectroscopy of UV-vis, FTIR, fluorescence, atomic force microscopy (AFM) and transmission electron microscopy (TEM).

\section{Characterization of human insulin fibrillation}

Human insulin was dissolved in hydrochloric acid aqueous solution ( $\mathrm{pH} 1.6$ ) with $100 \mathrm{Mm}$ sodium chloride to a concentration of $1 \mathrm{mg} / \mathrm{mL}$ and incubated at $37^{\circ} \mathrm{C}$ with stirring in a sand bath [27]. Insulin/DHLA-CDs mixture samples were also prepared at the same concentration of protein in the presence of 0.2 or $2 \mu \mathrm{g} / \mathrm{mL}$ of DHLACDs. Aliquots of samples were withdrawn at various time periods and characterized for fibril formation with the following methods.

Thioflavin $\mathrm{T}$ (ThT) fluorescence was recorded on a Fluorolog-3 spectrofluorometer at an excitation wavelength of $450 \mathrm{~nm}$ in a quartz cuvette with $1 \mathrm{~cm}$ optical path length. Aliquots of protein solutions were diluted with ThT solution to $0.1 \mathrm{mg} / \mathrm{mL}$ of protein and $20 \mu \mathrm{M}$ of ThT. In all measurements of fluorescence, emission and excitation slit widths were set at $5 \mathrm{~nm}$. The experiments were repeated three times and average emission intensity at $485 \mathrm{~nm}$ was plotted against incubation time.

Circular dichroism spectra were obtained on a JASCO J-810 spectropolarimeter (Easton, MD). The spectra were recorded between 190 and $260 \mathrm{~nm}$ at room temperature employing a $2 \mathrm{~mm}$ optical path length quartz cell. The spectra were measured using diluted aliquots (to $0.1 \mathrm{mg} / \mathrm{mL}$ of insulin) withdrawn from the human insulin or insulin/DHLA-CDs mixture solutions.

Atomic force microscopy images were taken with tapping mode by an Agilent 5420 AFM instrument (Santa Clara, CA) with a resolution of $512 \times 512$ pixels. The cantilever had a resonance frequency of $\sim 170 \mathrm{kHz}$ with typical force constant of $7.5 \mathrm{~N} / \mathrm{m}$. The sample was drop-coated on a freshly cleaved mica slide and allowed to dry. Then the sample coated mica slide was gently washed with pure water to remove the salt and allowed to air dry. Transmission electron microscopy (TEM) image of DHLA-CDs was obtained on a JEOL 1200X TEM.

\section{Results and Discussion}

\section{Characterization of hydrophobic CDs and DHLA-CDs}

The hydrophobic carbon dots were synthesized using the procedure reported by Wang et al. [25]. The as-prepared hydrophobic carbon dots are soluble in chloroform and highly fluorescent under $365 \mathrm{~nm}$ UV lamp (Figure 1A). The UV-vis absorption spectrum contains a peak at about $360 \mathrm{~nm}$ (Figure 1B). The maximal fluorescence emission peak of these carbon dots is dependent on the excitation wavelength (Figures $1 \mathrm{C}$ and $1 \mathrm{D}$ ), and it shifts toward higher wavelengths when excited at larger wavelengths. The size of the prepared hydrophobic CDs is about 6-8 $\mathrm{nm}$ based on atomic force microscopy imaging. All these characterizations are consistent with the results reported before [25].

CDs were conjugated with DHLA to increase their water solubility. After preparation, the DHLA-CDs were characterized by FTIR, UV-Vis, fluorescence and AFM (Figure 2). As displayed in Figure 2A, the absorbance peaks of the carboxyl group at $1705 \mathrm{~cm}^{-1}$ in CDs and DHLA vanished after conjugation, while a weak peak around $1017 \mathrm{~cm}^{-1}$ appeared in DHLA-CDs. These observations suggest the formation of a thioester. Compared with CDs in chloroform, the UV-vis absorption peak of DHLA-CDs in water becomes weaker and blue shifted to about $325 \mathrm{~nm}$ (Figure 2B). In addition, the maximal fluorescence intensity of DHLA-CDs in aqueous solution becomes much smaller after conjugation compared to that of CDs in chloroform at the same concentration (i.e. $0.15 \mathrm{mg} / \mathrm{mL}$, Figure 2C). It is worth noting that the sharp peaks in Figure $2 \mathrm{C}$ are caused by the Raman scattering. The fluorescence emission peak is the broad one neighboring the Raman scattering peaks. Compared to the strong emission of the core (Figure 1C), the reduced fluorescence of DHLA-CDs is likely due to the presence of DHLA outside the surface of the hydrophobic core of CDs, modifying the surface passivation of CDs. The atomic force microscopy (AFM) image in Figure 2D illustrates the morphology of CDs after conjugation, which shows the 
Citation: Kuruvilla SJ, Li S, Sansalone L, Fortes B, Zheng I, et al. Dihydrolipoic Acid Conjugated Carbon Dots Accelerate Human Insulin Fibrillation. J Parkinsons Dis Alzheimer Dis. 2015;2(1): 7.

(A)

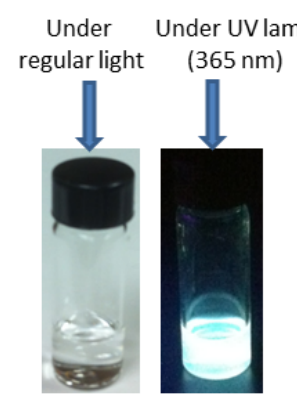

(C)

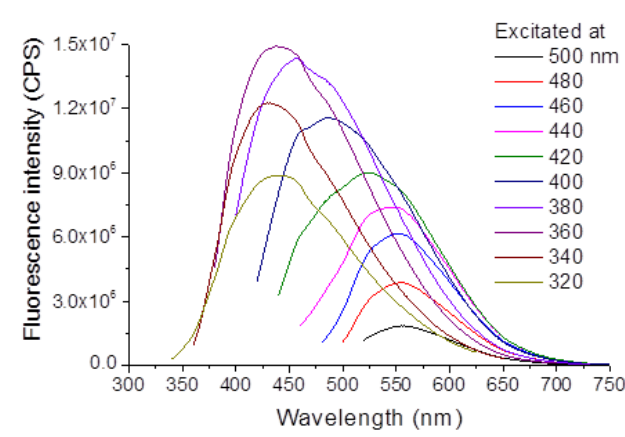

(B)

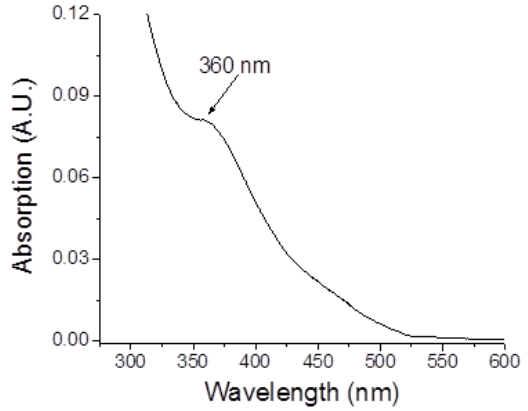

(D)

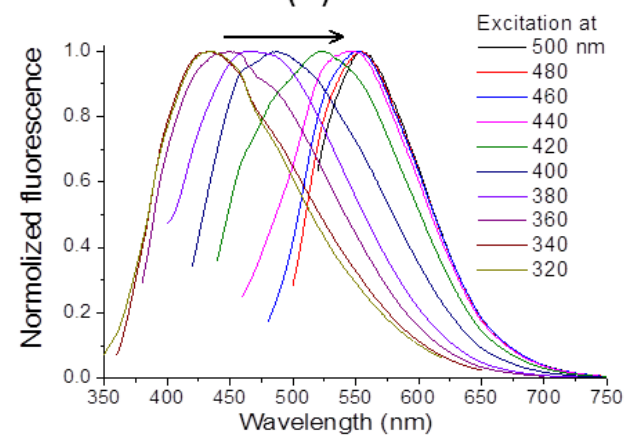

Figure 1: (A) Hydrophobic carbon dots dissolved in chloroform under regular light or $365 \mathrm{~nm}$ UV lamp; (B) UV-vis absorption of hydrophobic carbon dots at 0.8 $\mathrm{mg} / \mathrm{mL}$ in chloroform; (C) Fluorescence spectra of hydrophobic carbon dots at $0.15 \mathrm{mg} / \mathrm{mL}$ in chloroform under various excitation wavelengths; (D) Normalized fluorescence spectra.

(A)

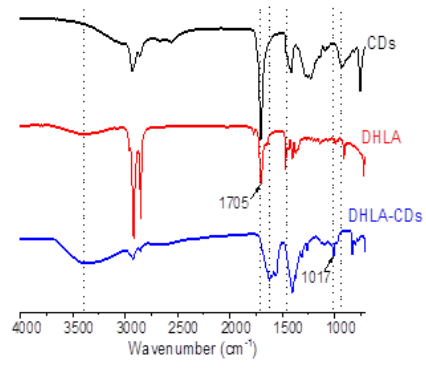

(D)

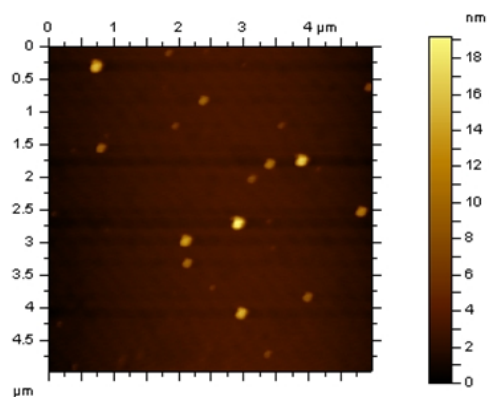

(B)
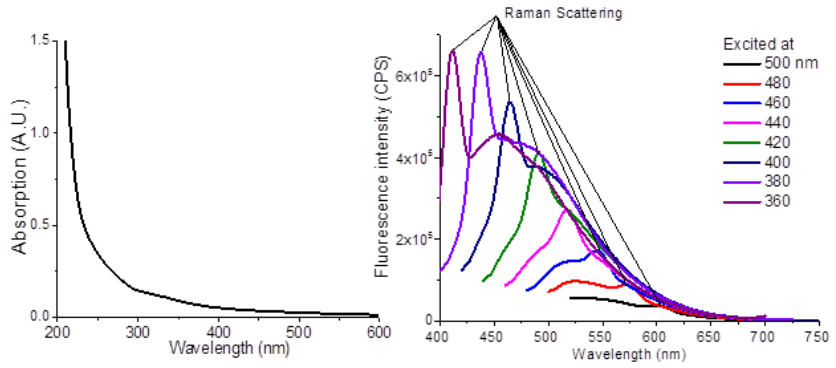

(E)

Figure 2: (A) FTIR spectra of hydrophobic carbon dots (CDs), DHLA, and DHLA conjugated CDs (DHLA-CDs); (B) UV-vis absorption of DHLA-CDs in water; (C) Fluorescence emission spectra of $0.15 \mathrm{mg} / \mathrm{mL}$ DHLA-CDs in water (i.e. the broad peaks neighboring the Raman scattering) under various excitation wavelengths; (D) AFM and (E) TEM image of DHLA-CDs. 
Citation: Kuruvilla SJ, Li S, Sansalone L, Fortes B, Zheng I, et al. Dihydrolipoic Acid Conjugated Carbon Dots Accelerate Human Insulin Fibrillation. J Parkinsons Dis Alzheimer Dis. 2015;2(1): 7.

height profile ranging from 12 to $20 \mathrm{~nm}$. The transmission electron microscopy (TEM) image in Figure 2E displays that the sizes of the particles are between 10 to $30 \mathrm{~nm}$.

\section{Thioflavin $\mathrm{T}$ fluorescence of human insulin incubated with DHLA-CDs}

Human insulin solutions were incubated at a constant insulin concentration of $1 \mathrm{mg} / \mathrm{mL}$ in $100 \mathrm{mM} \mathrm{NaCl}$ solution at $\mathrm{pH} 1.6$ in the absence or presence of DHLA-CDs at $37^{\circ} \mathrm{C}$. It has been previously reported that human insulin is able to form fibrils in vitro under these ionic strength, $\mathrm{pH}$ and temperature conditions [27]. Similar to the fibrillation of other proteins, it is proposed that human insulin undergoes its fibrillation process through a three-state transition: (1) a lag phase, (2) an initiation stage where fibril formation starts taking place and increases rapidly with time and (3) the final saturation phase which features a plateau of mature fibril formation $[28,29]$. Figure 3 exhibits thioflavin T (ThT) fluorescence of human insulin in the absence and presence of DHLA-CDs (i.e. $0,0.2$ and $2 \mu \mathrm{g} / \mathrm{mL}$ ). The intensity of fluorescence emission of ThT at $485 \mathrm{~nm}$ increases with incubation time, indicating the formation of insulin fibrils [3032]. All the samples containing human insulin show an increase in fluorescence intensity with the time of incubation, while $2 \mu \mathrm{g} /$ $\mathrm{mL}$ DHLA-CDs alone does not display ThT fluorescence intensity increase at $485 \mathrm{~nm}$ with the time of incubation. For the insulin sample (black curve in Figure 3), a well-defined lag time is observed where there is no significant increase in $\mathrm{ThT}$ fluorescence intensity at 485 $\mathrm{nm}$ for the first 4 hours of incubation. After the first 4 hours, a gradual increase in intensity is observed, which reaches a plateau after about 6 hours of incubation. However, in the presence of $0.2 \mathrm{or} 2 \mu \mathrm{g} / \mathrm{mL}$ of DHLA-CDs, an increase of fluorescence intensity at $485 \mathrm{~nm}$ is observed at the beginning (red and blue curves in Figure 3). The lag phase time is significantly reduced in the presence of DHLA-CDs. At each time period of incubation, higher concentrations of DHLA-CDs have greater ThT fluorescence intensity, suggesting that the presence of more DHLA-CD results in a higher rate of insulin fibrillation.

\section{Secondary conformational changes of human insulin incubated with DHLA-CDs}

Circular dichroism is a spectroscopic technique widely used to characterize conformational changes of proteins and peptides [33-35]. In the experiment, the conformational changes of human insulin in the absence and presence of DHLA-CDs were monitored with circular dichroism spectroscopy during the fibrillation process. Figures $4 \mathrm{~A}-4 \mathrm{C}$ shows the spectra of aliquots withdrawn at incubation times of $0,3.5$ and 6 hours, respectively. In the absence and presence of DHLA-CDs, human insulin at time 0 has almost exactly the same circular dichroism spectra with two negative bands at 208 and 222 $\mathrm{nm}$, which are characteristics of the $\alpha$-helix conformation (Figure 4A). After 3.5 hours of incubation, the peak at $208 \mathrm{~nm}$ becomes weaker and a broad band around $218 \mathrm{~nm}$ begins to appear, indicating that the secondary conformation changes from $\alpha$-helix to $\beta$-sheet (Figure 4B). It is worth noting that human insulin at time 3.5 hours in the presence of DHLA-CDs experiences a larger conformational change than that in the absence of DHLA-CDs based on the ellipticity at $218 \mathrm{~nm}$. After 6 hours of incubation, human insulin changes to $\beta$-sheet in all cases (Figure 4C).

Circular dichroism spectra in Figure 4 indicate that all human insulin samples are transformed to a $\beta$-sheet conformation with increasing time of incubation. However, the rate of this transformation increases in the presence of DHLA-CDs. Therefore, the circular dichroism spectra illustrate that the rate of insulin's $\beta$-sheet transformation takes place in a shorter amount of time when DHLA-CDs are present.

\section{AFM imaging analysis}

AFM images of samples were analyzed at 30 minutes time intervals throughout the incubation process to monitor progress of fibril formation. Figure 5 displays the AFM images of samples at incubation time intervals of $0,0.5,3.5$ and 6 hours. At time 0 , all three samples exhibit no fibril formation. The next sample was taken at 0.5

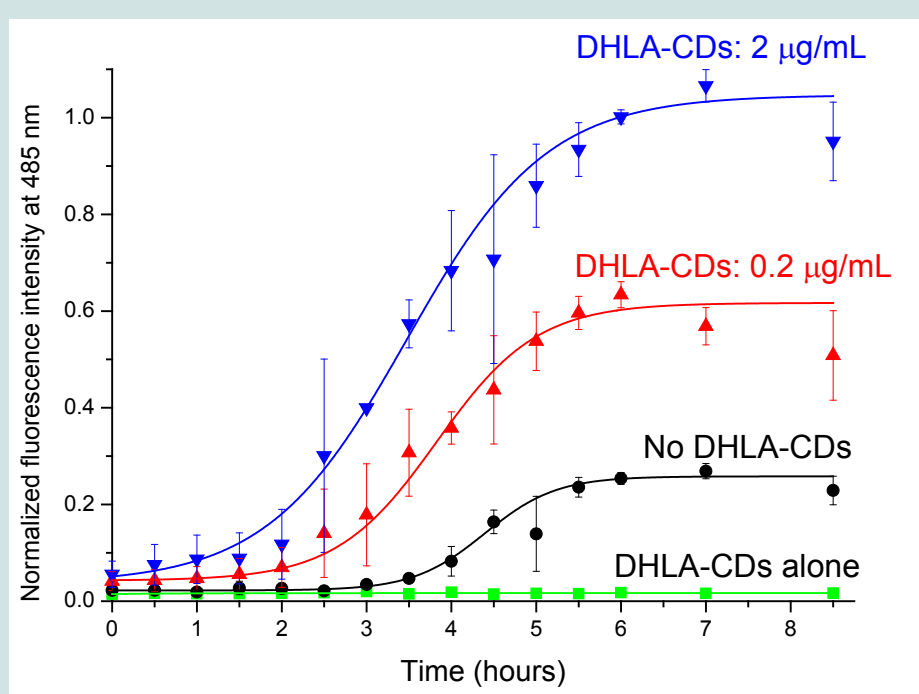

Figure 3: Normalized thioflavin T (ThT) fluorescence intensity at $485 \mathrm{~nm}$ of 10 -fold diluted samples against incubation time in the absence and presence of DHLACDs. The original samples contained $1 \mathrm{mg} / \mathrm{mL}$ of human insulin with 0 (black), 0.2 (red) or 2 (blue) $\mu \mathrm{g} / \mathrm{mL}$ of DHLA-CDs. The green curve is a control experiment with $2 \mu \mathrm{g} / \mathrm{mL}$ of DHLA-CDs mixed with ThT. The excitation wavelength was $450 \mathrm{~nm}$. 
Citation: Kuruvilla SJ, Li S, Sansalone L, Fortes B, Zheng I, et al. Dihydrolipoic Acid Conjugated Carbon Dots Accelerate Human Insulin Fibrillation. J Parkinsons Dis Alzheimer Dis. 2015;2(1): 7.

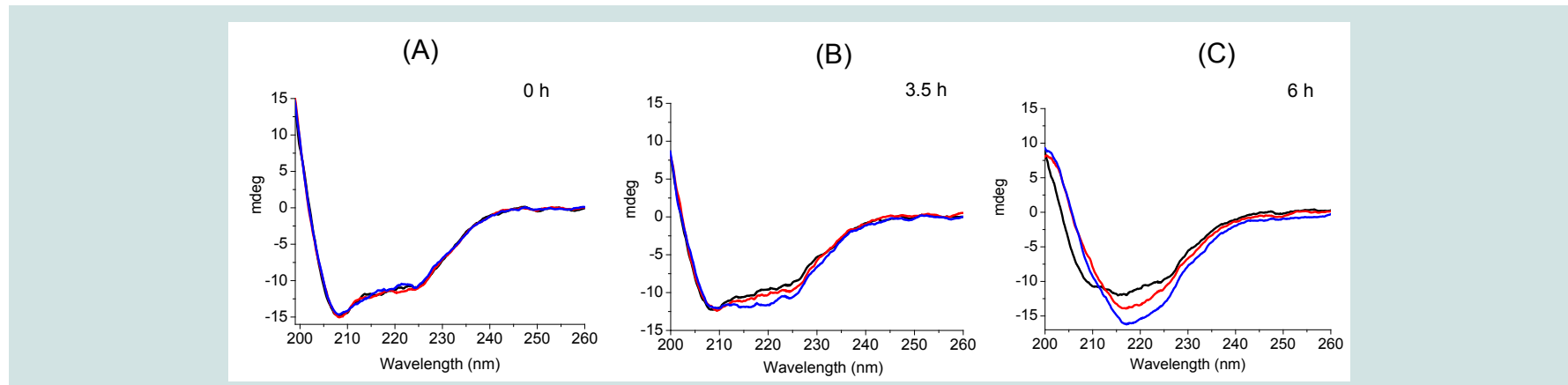

Figure 4: Circular dichroism spectra of human insulin incubated in the absence and presence of DHLA-CDs for (A) 0 , (B) 3.5 and (C) 6 hours. The original samples contained $1 \mathrm{mg} / \mathrm{mL}$ of human insulin with 0 (black), 0.2 (red) or $2 \mu \mathrm{g} / \mathrm{mL}$ (blue) of DHLA-CDs. The concentration of human insulin was diluted to $0.1 \mathrm{mg} / \mathrm{mL}$ in all cases to obtain circular dichroism spectra. It is worth noting that the whole circular dichroism spectrum of $2 \mu \mathrm{g} / \mathrm{mL}$ DHLA-CDs alone is around 0 (data not shown).

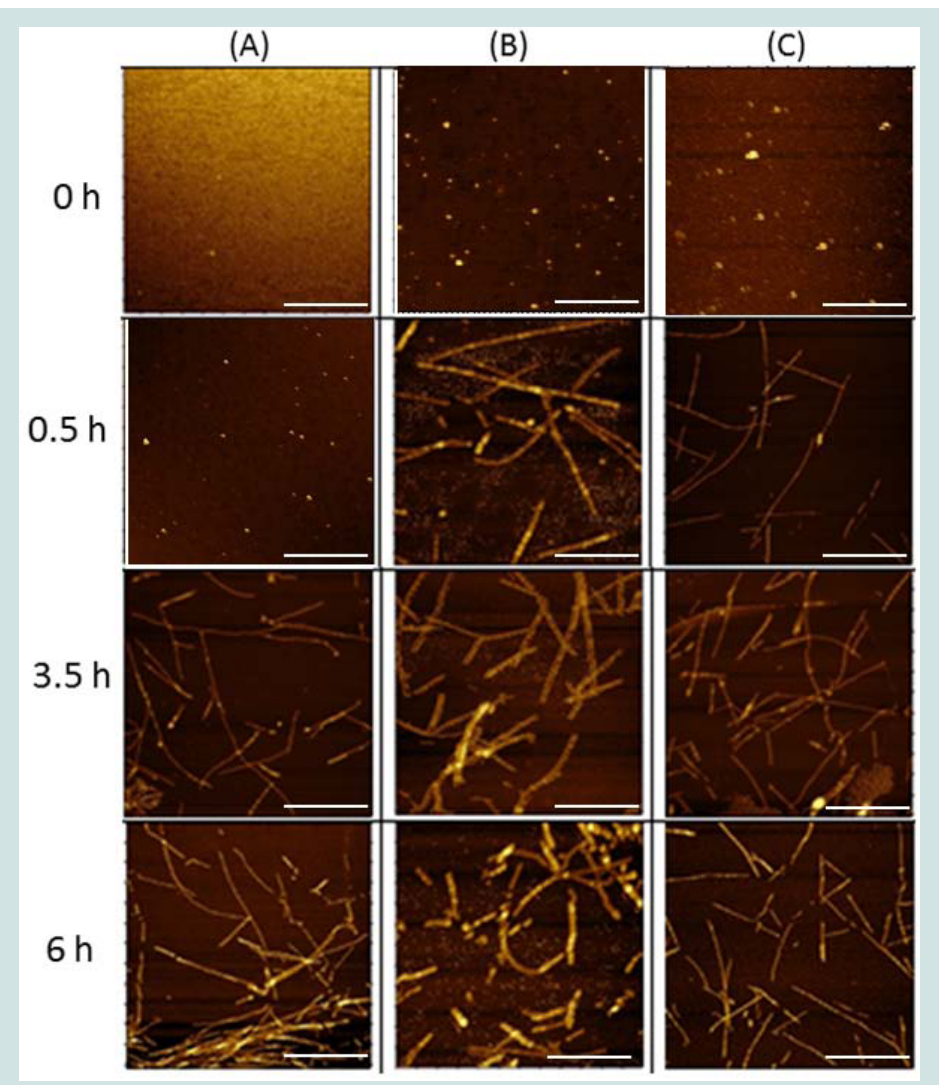

Figure 5: AFM images of samples at incubation times of $0,0.5,3.5$ and 6 hours: (A) insulin in the absence of DHLA-CDs; (B) insulin with $0.2 \mu \mathrm{g} / \mathrm{mL}$ of DHLA-CDs: (C) insulin with $2 \mu \mathrm{g} / \mathrm{mL}$ of DHLA-CDs. The scale bar shows $1 \mu \mathrm{m}$.

hours after incubation at $37^{\circ} \mathrm{C}$. The topography of the human insulin sample in the absence of DHLA-CDs looks similar to that at time 0. This sample does not show the formation of fibrils until 3.5 hours of incubation (Figure 5A). However, insulin fibrils are observed after 30 minutes of incubation in the presence of DHLA-CDs with height $10 \sim 20 \mathrm{~nm}$ and length $0.5 \sim 2 \mu \mathrm{m}$ (Figures 5B-5C). Based on these observations, protofibrils are expected to form during the first 30 minutes of incubation in the presence of DHLA-CDs. From AFM images, it is difficult to judge whether the proto fibrils or fibrils bind to or grow from the particles of DHLA-CDs. All three samples were next measured at 6 hours and they all demonstrated the presence of fibrils.
Therefore, the AFM images also confirm that the presence of DHLACDs increases the rate of human insulin fibrillation by shortening the lag phase time. Mature insulin fibrils are observed within 30 minutes of incubation in the presence of DHLA-CDs, whereas it takes about 3.5 hours for insulin fibrils to appear in the absence of DHLA-CDs.

\section{Discussion}

It is widely accepted that the typical protein fibrillation process involves a number of intermediate states, including nucleation, oligomerization, and fibril formation $[3,28,29,36]$. The formation of the nucleation species (also called lag phase) is the key rate-determining 
Citation: Kuruvilla SJ, Li S, Sansalone L, Fortes B, Zheng I, et al. Dihydrolipoic Acid Conjugated Carbon Dots Accelerate Human Insulin Fibrillation. J Parkinsons Dis Alzheimer Dis. 2015;2(1): 7.

step, as it is a thermodynamically unfavored process during protein fibrillation. It has been known recently that the presence of nanoparticles can significantly affect protein conformation as a result of the interactions between nanoparticles and proteins $[12,14,15,21]$. Therefore, it is not unexpected that the presence of nanoparticles may accelerate or inhibit protein fibrillation. Although extensive studies are now being carried out on the effects of nanoparticles on protein fibrillation, our current knowledge still relies on a case-bycase approach [37]. In general, the effects are due to the interaction between proteins and the surface of nanoparticles, depending on the protein surface properties (charge, stability, hydrophobicity), and on the nanoparticle characteristics (charge, size, coating, components) $[12,14,15,37]$.

In the present study, an accelerating effect on human insulin fibrillation, by shortening the lag phase time, was observed in the presence of DHLA-CDs. Although the detailed molecular mechanism of nucleation on the surface of these nanoparticles remains elusive, the fibrillation process is believed to be associated with the locally increased concentration of insulin in the vicinity of the nanoparticle surface due to the adsorption [12,38-40]. It is worth noting that the adsorption is likely from weak interactions, such as hydrophobic interaction, hydrogen bonding and electrostatic interaction. Among them, the major contributor is expected to be the hydrophobic interaction between human insulin and the surface of DHLA-CDs. The surface of the core of CDs and the carbon chain of DHLA are both hydrophobic, providing a hydrophobic interface for the insulin adsorption. Similar to the scenario of insulin at a hydrophilic-hydrophobic interface, the insulin monomers unfold the native conformations followed by the adsorption [3,41-43]. This process facilitates the subsequent assembly of disordered oligomeric structures. Thereafter, these assemblies undergo a process of reordering into $\beta$-sheet structures, which are driven by hydrogen bonding formation [40].

The enhanced fibrillation effect was more obvious at higher concentrations of DHLA-CDs under the same concentration of human insulin. Parameters of nanoparticles under this condition were the same, such as surface charge, coating, and surface curvature. The main difference may come from the total surface area available on DHLA-CDs for insulin adsorption. Upon adsorption, the insulin monomers unfold the conformations, which facilitate the formation of insulin oligomers. At higher concentrations of DHLA-CDs, larger surface areas are available for unfolding insulin monomers, thus generating more oligomers. The continuing assembly of oligomers will produce more nuclei. Once the critical concentration of nucleation is reached, elongation of insulin fibrils will rapidly occur.

Most recently we reported that water soluble fluorescent CDs with an average diameter of $4 \mathrm{~nm}$ were prepared by a "top-down" approach from oxidizing carbon nanopowder. Our experiments demonstrated that these CDs strongly inhibit human insulin fibrillation in a concentration-dependent manner [21]. Increasing the concentration of CDs enhances the inhibiting effect. On the contrary, the presence of the DHLA-CDs in this study promotes human insulin fibrillation in a concentration-dependent manner. One has to point out that the DHLA-CDs have completely different nature in terms of size, surface property and component from those we reported in Reference [21]. As displayed in Figure 2D and 2E, the size of the DHLA-CDs is much larger (i.e. $10 \sim 30 \mathrm{~nm}$ ) than those water soluble fluorescent CDs. Strictly speaking, the DHLA-CDs should not be called carbon dots any more due to the large size. Furthermore, the DHLA-CDs have a hydrophobic core capped by dihydrolipoic acid which has a long nonpolar carbon chain. On the other hand, the surface of the CDs used in Reference [21] is highly hydrophilic with plenty of carboxylic groups. To summarize, we believe that these differences of size, surface property and component determine the opposite observations on human insulin fibrillation. As mentioned in the introduction, our current knowledge of nanoparticles influencing protein fibrillation still relies on a case-by-case approach $[12,14,15]$.

\section{Conclusions}

Protein fibrillation has been found to play a significant role in several human diseases, such as Alzheimer's disease, Parkinson's disease and type 2 diabetes. Some nanoparticles have been shown to have potential applications in nanomedicine and biomedical engineering due to their interactions with proteins. Carbon dots, as a new type of nanoparticle, have been extensively studied for their applications in biological systems, but their effects on protein fibrillation remain elusive. In this study, DHLA-conjugated carbon dots were synthesized by capping the hydrophobic core with dihydrolipoic acid. Their effect on human insulin fibrillation was investigated in vitro by incubation at $37^{\circ} \mathrm{C}$. The experiments illustrate that the presence of DHLA-CDs results in a higher rate of human insulin fibrillation, accelerating the secondary conformational change of human insulin from $\alpha$-helix to $\beta$-sheet. This promoting effect of DHLA-CDs may be associated with the locally increased concentration of human insulin adsorbed on their surface and conformational changes after adsorption.

\section{References}

1. Chiti F, Dobson CM (2006) Protein misfolding, functional amyloid, and human disease. Annu Rev Biochem 75: 333-366.

2. Nelson R, Eisenberg D (2006) Recent atomic models of amyloid fibril structure. Curr Opin Struct Biol 16: 260-265.

3. Li S, Leblanc RM (2014) Aggregation of insulin at the interface. J Phys Chem B 118: 1181-1188.

4. Ahmad A, Uversky VN, Hong D, Fink AL (2005) Early events in the fibrillation of monomeric insulin. J Biol Chem 280: 42669-42675.

5. Sunde M, Serpell LC, Bartlam M, Fraser PE, Pepys MB, et al. (1997) Common core structure of amyloid fibrils by synchrotron X-ray diffraction. J Mol Biol 273: 729-739.

6. Prado-Gotor R, Grueso E (2011) A kinetic study of the interaction of DNA with gold nanoparticles: mechanistic aspects of the interaction. Phys Chem Chem Phys 13: 1479-1489.

7. Cedervall T, Lynch I, Lindman S, Berggård T, Thulin E, et al. (2007) Understanding the nanoparticle-protein corona using methods to quantify exchange rates and affinities of proteins for nanoparticles. Proc Natl Acad Sci U S A 104: 2050-2055.

8. Mironava T, Hadjiargyrou M, Simon M, Jurukovski V, Rafailovich MH (2010) Gold nanoparticles cellular toxicity and recovery: Effect of size, concentration and exposure time. Nanotoxicology 4: 120-137

9. Li S, Mulloor JJ, Wang L, Ji Y, Mulloor CJ, et al. (2014) Strong and selective adsorption of lysozyme on graphene oxide. ACS Appl Mater Interfaces 6: 5704-5712.

10. Li S, Stein AJ, Kruger A, Leblanc RM (2013) Head groups of lipids govern the 
Citation: Kuruvilla SJ, Li S, Sansalone L, Fortes B, Zheng I, et al. Dihydrolipoic Acid Conjugated Carbon Dots Accelerate Human Insulin Fibrillation. J Parkinsons Dis Alzheimer Dis. 2015;2(1): 7.

interaction and orientation between graphene oxide and lipids. J Phys Chem C 117: 16150-16158.

11. Xiao L, Zhao D, Chan WH, Choi MM, Li HW (2010) Inhibition of beta 1-40 amyloid fibrillation with $\mathrm{N}$-acetyl-I-cysteine capped quantum dots. Biomaterials 31: $91-98$.

12. Linse S, Cabaleiro-Lago C, Xue WF, Lynch I, Lindman S, et al. (2007) Nucleation of protein fibrillation by nanoparticles. Proc Natl Acad Sci U S A 104: 8691-8696

13. Vannoy CH, Leblanc RM (2010) Effects of DHLA-capped CdSe/ZnS quantum dots on the fibrillation of human serum albumin. J Phys Chem B 114: 10881 10888.

14. Mahmoudi M, Kalhor HR, Laurent S, Lynch I (2013) Protein fibrillation and nanoparticle interactions: opportunities and challenges. Nanoscale 5: 25702588.

15. Yoo SI, Yang M, Brender JR, Subramanian V, Sun K, et al. (2011) Inhibition of amyloid peptide fibrillation by inorganic nanoparticles: functional similarities with proteins. Angew Chem Int Ed 50: 5110-5115.

16. Yang ST, Wang X, Wang H, Lu F, Luo PG, et al. (2009) Carbon dots as nontoxic and high-performance fluorescence imaging agents. J Phys Chem C 113: 18110-18114.

17. Ruan S, Qian J, Shen S, Zhu J, Jiang X, et al. (2014) A simple one-step method to prepare fluorescent carbon dots and their potential application in non-invasive glioma imaging. Nanoscale 6: 10040-10047.

18. Xu X, Ray R, Gu Y, Ploehn HJ, Gearheart L, et al. (2004) Electrophoretic analysis and purification of fluorescent single-walled carbon nanotube fragments. J Am Chem Soc 126: 12736-12737.

19. Tao H, Yang K, Ma Z, Wan J, Zhang Y, et al. (2012) In vivo NIR fluorescence imaging, biodistribution, and toxicology of photoluminescent carbon dots produced from carbon nanotubes and graphite. Small 8: 281-290.

20. Huang YF, Zhou X, Zhou R, Zhang H, Kang KB, et al. (2014) One-po synthesis of highly luminescent carbon quantum dots and their nontoxic ingestion by zebrafish for in vivo imaging. Chemistry 20: 5640-5648.

21. Li S, Wang L, Chusuei CC, Suarez VM, Blackwelder PL, et al. (2015) Nontoxic carbon dots potently inhibit human insulin fibrillation. Chem Mater 27: 17641771.

22. Yumlu S, Barany R, Eriksson M, Röcken C (2009) Localized insulin-derived amyloidosis in patients with diabetes mellitus: a case report. Hum Pathol 40 1655-1660.

23. Skaat H, Belfort G, Margel S (2009) Synthesis and characterization of fluorinated magnetic core-shell nanoparticles for inhibition of insulin amyloid fibril formation. Nanotechnology 20: 225106.

24. Jiménez JL, Nettleton EJ, Bouchard M, Robinson CV, Dobson CM, et al. (2002) The protofilament structure of insulin amyloid fibrils. Proc Natl Acad Sci U S A 99: 9196-9201.

25. Wang F, Pang S, Wang L, Li Q, Kreiter M, et al. (2010) One-step synthesis of highly luminescent carbon dots in noncoordinating solvents. Chem Mater 22 : 4528-4530.

26. Clapp AR, Goldman ER, Mattoussi H (2006) Capping of CdSe-ZnS quantum dots with DHLA and subsequent conjugation with proteins. Proc Natl Acad
Sci U S A 1: 1258-1266.

27. Groenning M, Frokjaer S, Vestergaard B (2009) Formation mechanism of insulin fibrils and structural aspects of the insulin fibrillation process. Curr Protein Pept Sci 10: 509-528.

28. Hua QX, Weiss MA (2004) Mechanism of insulin fibrillation: the structure of insulin under amyloidogenic conditions resembles a protein-folding intermediate. J Biol Chem 279: 21449-21460.

29. Sluzky V, Klibanov AM, Langer R (1992) Mechanism of insulin aggregation and stabilization in agitated aqueous solutions. Biotechnol Bioeng 40: 895903.

30. Ban T, Hamada D, Hasegawa K, Naiki H, Goto Y (2003) Direct observation of amyloid fibril growth monitored by thioflavin T fluorescence. J Biol Chem 278 : 16462-16465.

31. Krebs MR, Bromley EH, Donald AM (2005) The binding of thioflavin-T to amyloid fibrils: localisation and implications. J Struct Biol 149: 30-37.

32. Sessions K, Sacks S, Li S, Leblanc RM (2014) epi-Fluorescence imaging at the air-water interface of fibrillization of bovine serum albumin and human insulin. Chem Commun 50: 8955-8957.

33. Greenfield NJ (2007) Using circular dichroism spectra to estimate protein secondary structure. Nat Protocols 1: 2876-2890.

34. Li S, Potana S, Keith DJ, Wang C, Leblanc RM (2014) Isotope-edited FTIR in $\mathrm{H} 2 \mathrm{O}$ : determination of the conformation of specific residues in a model a-helix peptide by $13 \mathrm{C}$ labeled carbonyls. Chem Commun 50: 3931-3933.

35. Matsuo K, Sakurada Y, Yonehara R, Kataoka M, Gekko K (2007) Secondarystructure analysis of denatured proteins by vacuum-ultraviolet circular dichroism spectroscopy. Biophys J 92: 4088-4096.

36. Kelly JW (1998) The alternative conformations of amyloidogenic proteins and their multi-step assembly pathways. Curr Opin Struct Biol 8: 101-106.

37. Colvin V L, Kulinowski KM (2007) Nanoparticles as catalysts for protein fibrillation. Proc Natl Acad Sci U S A 104: 8679-8680.

38. Lynch I, Dawson KA (2008) Protein-nanoparticle interactions. Nano Today 3 : 40-47.

39. Wu WH, Sun X, Yu YP, Hu J, Zhao L, et al. (2008) TiO2 nanoparticles promote $\beta$-amyloid fibrillation in vitro. Biochem Biophys Res Commun 373: 315-318.

40. Auer S, Trovato A, Vendruscolo M (2009) A Condensation-ordering mechanism in nanoparticle-catalyzed peptide aggregation. PLoS Comput Biol 5: e1000458.

41. Thurow H, Geisen K (1984) Stabilisation of dissolved proteins against denaturation at hydrophobie interfaces. Diabetologia 27: 212-218.

42. Kwon YM, Baudys M, Knutson K, Kim SW (2001) In situ study of insulin aggregation induced by water-organic solvent interface. Pharm Res 18: 1754-1759

43. Donsmark J, Jorgensen L, Mollmann S, Frokjaer S, Rischel C (2006) Kinetics of insulin adsorption at the oil-water interface and diffusion properties of adsorbed layers monitored using fluorescence correlation spectroscopy. Pharm Res 23: 148-155.

\section{Acknowledgement}

This study was supported by National Science Foundation under Grant 1355317. 